\title{
Treatment of prostate cancer by hypofractionated radiotherapy
}

\author{
Sefik IGDEM
}

\begin{abstract}
Technological advances in radiation therapy delivery have permitted the use of high dose per fraction radiotherapy for early stage prostate cancer. Level I evidence supporting the safety and efficasy of hypofractionated radiotherapy is evolving. Moderate and extreme hypofractionation regimens show promising cancer control but long term results of noninferiority studies of both modalities are required before use in routine treatment as standard of care.
\end{abstract}

Keywords: Prostate cancer, Curative radiotherapy, Moderate and extreme hypo fractionation

\section{Introduction}

a good therapeutic outcome in radiotherapy comprises both local control of the tumor and minimal late normal tissue complications. Recent advances in radiotherapy technologies have sparked interest in hypofractionation utilizing targeting to minimize normal tissue injury rather than dose modulation. Beyond biology, hypofractionation for prostate cancer has the advantages of increased convenience for the patient and a lower cost burden for the health care system.

Curative radiotherapy treatment regimens involve dividing the overall radiation dose into a number of fractions, as this takes advantage of the five Rs of radiotherapy:

Sefik Igdem, M.D. (四)

Department of Radiation Oncology, Medical School, Istanbul Bilim University Istanbul, Turkey

e-mail: sefikigdem@gmail.com reoxygenation of hypoxic cells, repopulation of cells, repair of normal tissue, redistribution of cells through the cell cycle and differences in the intrinsic radiosensitivity of tumor cells compared with normal tissue cells. A typical conventional fractionation regimen uses 2 Gy daily doses delivered five times a week, up to a total of 60-80 Gy. A good therapeutic outcome in radiotherapy comprises both local control of the tumor and minimal late normal tissue complications. As the dose per fraction increases, the probability of toxic effects in late responding normal tissue increases disproportionately compared with early responding normal tissues, which are typically less sensitive to fractionation. It is primary late responding normal tissue toxicity that typically limits radiotherapy treatment regimens.

Recent advances in radiotherapy technologies have sparked interest in hypofractionation, a highly conformal radiotherapy delivering daily doses of 2.5-10 Gy, utilizing targeting to minimize normal tissue injury rather than dose modulation. The biologic rationale for applying hypofractionation to prostate cancer is based on the theory that the slow proliferation of prostate cancer cells leads to a biologic radiation response in prostate cancer that differs from most other cancers. Traditional fractionation causes the accumulation of DNA damage, ultimately causing apoptosis, mitotic catastrophe, or senescence. A slow proliferation rate results in a high reparation ability of radiation damage over time, such that standard fractionation given in small increments over a long time period may be suboptimal for prostate cancer for which a high total dose is required for effective control. For slowly proliferating cells high doses per fraction may be more effective because immediate cell death is instigated due to high number of DNA double strand breaks caused by each fraction. 
Beyond biology, hypofractionation for prostate cancer has the advantages of increased convenience for the patient and a lower cost burden for the health care system.

Hypofractionation has arisen in the setting of an increased ability to plan and target radiotherapy to a given target. In the case of curative radiotherapy of prostate cancer the use of intensity-modulated radiation therapy (IMRT) has allowed highly conformal treatment plans where the dose gradient is quite steep, allowing for reduced dose to nearby normal tissues. Image guided radiation therapy (IGRT) via ultrasound, $x$-ray, or computed tomography imaging of the prostate allows proper placement of the radiation fields each day. Most of the studies utilizing hypofractionation deliver the radiation dose using IMRT with image guidance, although several older studies were performed with 3D conformal radiotherapy. Extreme hypofractionation also known as stereotactic body radiation therapy (SBRT) is often delivered using a specialized linear accelerator on a robotic arm, but it can be delivered by any accelerator with IMRT and on board imager.

\section{Moderate hypofractionation}

There are many reports of moderate hypofractionation using external beam radiotherapy, with doses per fraction varying from 2.5 to $3.16 \mathrm{~Gy}$. The largest of these is the CHHiP study, which randomized patients between 2 Gy per fraction (to 74 Gy in 37 fractions) or hypofractionated (60 Gy in 20 fractions or $57 \mathrm{~Gy}$ in 19 fractions) IMRT [1]. This trial has recruited over 3000 men and has now published data from interim safety analysis of the first 457 patients randomized. Late toxicity measured at two years after treatment was low for all arms. RTOG grade $\geq 2$ gastrointestinal toxicity seen in $4.3,3.6$ and $1.4 \%$ in the 74,60 , and 57 Gy arms, respectively. Acute toxicity of RTOG grade 2 or higher was seen in $0.8-2.3 \%$ (GI) and 7-7.6\% (GU). Acute toxicity had a brisker onset and a higher peak for the hypofractionated arms compared with the conventional fractionation. Acute toxicity was also noted to return to baseline more quickly in the experimental arms.

Arcangeli et al. [2] randomized 168 patients to $62 \mathrm{~Gy}$ in 20 fractions or $80 \mathrm{~Gy}$ in 40 fractions. Although the trial was small contemporary prescriptions and $3 \mathrm{D}$ conformal techniques were used, with all patients receiving 9 months of androgen suppression. Doses were iso-effective based on an alpha/beta ratio of 1.5 or 1.8 Gy (EQD2 81.5 or 79.9 Gy, respectively). The late toxicity was not significantly different between the hypofractionated and conventionally fractionated arms. (LENT-SOMA GI and GU toxicity $\geq$ grade 2 was 17 and $16 \%$ in the hypofractionated arm at 3 years compared with 14 and $11 \%$ in the conventional arm). Acute toxicity was acceptable and comparable with that of CHHiP. It was also noted that the hypofractionated arm had a nonsignificant increase in acute toxicity. The bRFS at 3 years was significantly improved in the hypofractionated arm, but the difference was marginal ( $87 \%$ vs $79 \%, p=0.035)[2]$ and no longer significant at 5 years $(85 \%$ vs $79 \%, \mathrm{p}=0.065)$ [3]. In addition the trial's primary end point was acute toxicity and, therefore was not adequately statistically powered to formally assess biochemical control.

Pollack et al. [4] reported the outcomes of the first 100 patients randomized to 76 Gy in 38 fractions versus $70.2 \mathrm{~Gy}$ in 26 fractions. No differences in maximal acute toxicity were seen, but a small increase in acute GI toxicity was noted during treatment. A 5-year update of this work showed no difference in biochemical outcomes [5]. The MD Anderson Cancer Center randomized 204 men to 75.6 Gy in 42 fractions versus $72 \mathrm{~Gy}$ in 30 fractions and found no significant difference in 5-year bRFS or toxicity [6].

Therefore the data from randomized trials to date can be interpreted as having iso-effective and iso-toxic late effects, with a possible marginal biochemical control benefit to biological dose escalation with moderate hypofractionation. As yet, moderate hypofractionation cannot be proven to be superior to conventional fractionation, but we can conclude that it seems to be safe and feasible without a significant increase in toxicity.

\section{Extreme hypofractionation}

Only phase 2 prospective studies of extreme hypofractionation with daily doses of 5-10 Gy to 35-50 Gy have been published, with follow up ranging from 18 months to 4.6 years. In general, these studies included only low risk and selected intermediate risk patients. In this patient population early cancer outcomes have been excellent. In the largest study with a follow up of 4.6 years Katz et al [7] treated 515 patients to 35-36.25 Gy in 5 fractions. Low risk patients had a freedom from biochemical failure rate of $97 \%$ at 6 years. Results for intermediate and high risk patients were 92\% and $70 \%$, respectively. Loblaw et al [8] treated only low risk prostate cancer patients and had a similar follow up of 55 months. The authors noted a 5-year biochemical control of $98 \%$. This study included posttreatment biopsies; $85 \%$ of the patients underwent biopsies with only $4 \%$ returning positive for residual cancer 3 years after treatment. Theses excellent results are early yet given the natural history of late recurrence in prostate cancer, but at 5 years they are similar to a high dose IMRT series for low risk disease [9].

Unfortunately, theses studies have not been randomized, 
and although supportive of the concept of hypofractionation, they cannot be used to direct routine care off protocol.

Moderate to high-grade acute toxicity from extreme hypofractionation ranges between $10 \%$ and $20 \%$ in these studies, with urinary symptoms more common than those relative to bowel and rectum. Late grade $\geq 2$ toxicity rates from these individual institutional experiences vary significantly (1-31\%), with occasional grade 4 toxicities reported. While urinary incontinence is uncommon in conventional fractionation, in one study it was reported as high as $10 \%$ in previously continent men 3 years post SBRT [10].

\section{Conclusion}

Given the demonstration thus far of at least comparable toxicities and biochemical outcomes for moderate hypofractionation, it is possible that with further evidence from maturing data, shorter regimens become the standard of care for prostate cancer. Whilst the evidence for extreme hypofractionation continues to accrue and with two randomized trials now recruiting, the clinical benefit, or otherwise, of SBRT will become clearer.

\section{References}

1. Dearnaley D, Syndikus I, Sumo G, et al. Conventional versus hypofractionated high dose intensity modulated radiotherapy for prostate cancer: preliminary safety results from the $\mathrm{CHHiP}$ randomised control trial. Lancet Oncol 2012;13:43-54. doi: 10.1016/S14702045(11)70293-5.

2. Arcangeli G, Saracino B, Gomellini S, et al. A prospective phase III randomised trial of hypofractination versus conventional fractionation in patients with high risk prostate cancer. Int $\mathrm{J}$ Radiat Oncol Biol Phys 2008;72:11-18. doi: 10.1016/j.ijrobp.2009.07.1691.
3. Arcangeli G, Strigari L, Gomellini S, et al. Updated results and patterns of failure in a randomized hypofractionation trial for high risk prostate cancer. Int $\mathrm{J}$ Radiat Oncol Biol Phys 2012;84:1172-8. doi: 10.1016/j. ijrobp.2012.02.049.

4. Pollack A, Hanlon AL, Horwitz EM, et al. Dosimetry and preliminary acute toxicity in the first 100 men treated for prostate cancer on a randomized hypofractionation dose escalation trial. Int $\mathrm{J}$ Radiat Oncol Biol Phys 2006;64:518-26.

5. Pollack A, Walker G, Buyyounouski M, et al. Five year results of a randomized external beam radiotherapy hypofractionation trial for prostate cancer. Int J Radiat Oncol Biol Phys 2011;81(Suppl. 2):S1

6. Kuban D, N.-G. GM, Hamblin L, et al. Preliminary report of a randomized dose escalation trial for prostate cancer using hypofractionation. Int J Radiat Oncol Biol Phys 2010;78(Suppl. 3):S58-S59.

7. Katz A, Kang J. Stereotactic body radiation therapy for low, intermediate and high risk prostate cancer: disease control and quality of life at 6 years. Int J Radiat Oncol Biol Phys 2013;87:524-5.

8. Loblaw A, Cheung P, D'Alimonte L, et al. Prostate stereotactic ablative body radiotherapy using a standard linear accelerator: toxicity, biochemical, and pathological outcomes. Radiother Oncol 2013;107:1538. doi: 10.1016/j.radonc.2013.03.022.

9. Alicikus ZA, Yamada Y, Zhang Z, et al. Ten year outcomes of high dose intensity modulated radiotherapy for localized prostate cancer. Cancer 2011;117:142937. doi: $10.1002 /$ cncr. 25467.

10. Chen LN, Suy S, Wang H, et al. Patient reported urinary incontinence following stereotactic body radiation therapy for clinically localized prostate cancer. Radiat Oncol 2014;9:148. doi: 10.1186/1748-717X-9-148. 